\title{
COMMUNICATION
}

Check for updates

Cite this: Chem. Commun., 2020 56, 13995

Received 28th August 2020,

Accepted 13th October 2020

DOI: $10.1039 / \mathrm{d} 0 \mathrm{cc} 05830 \mathrm{k}$

rsc.li/chemcomm

\section{Arylation of aryllithiums with $S$-arylphenothiazinium ions for biaryl synthesis $\dagger$}

\author{
Tatsuya Morofuji, (D)*a Tatsuki Yoshida, ${ }^{a}$ Ryosuke Tsutsumi, (DD ${ }^{b}$ \\ Masahiro Yamanaka (D) ${ }^{\mathrm{b}}$ and Naokazu Kano (D) *a
}

Aryllithiums are one of the most common and important aryl nucleophiles; nevertheless, methods for arylation of aryllithums to produce biaryls have been limited. Herein, we report arylation of aryllithiums with $S$-arylphenothiazinium ions through selective ligand coupling of intermediary sulfuranes. Various unsymmetrical biaryls could be obtained without transition-metal catalysis.

Aryllithiums are one of the most common and important aryl nucleophiles in organic chemistry. Aryllithiums are known to react with various electrophiles, such as alkyl halides, carbonyl compounds, chlorosilanes and trimethoxyborane. ${ }^{1}$ However, methods for arylation of aryllithums to produce biaryls are very limited. Arylation of aryllithiums based on nucleophilic aromatic substitution ${ }^{2}$ or nucleophilic attack to aryne $^{3}$ was reported, but there were few options regarding the applicable substrates. Although a few palladium-catalyzed methods have been reported, ${ }^{4}$ development of electrophilic arylation reagents that react with aryllithiums in the absence of transition metals is a simple but challenging problem (Fig. 1a). ${ }^{5}$

In sulfur chemistry, the ligand coupling of tetraarylsulfuranes (IUPAC nomenclature: tetraaryl- $\lambda^{4}$-sulfanes) has been reported. ${ }^{6}$ Reaction of triarylsulfoniums with arylmetal reagents including aryllithiums affords the tetraarylsulfurane intermediate, which decomposes to the corresponding biaryls and diaryl sulfides without transition-metal catalysis (Fig. 1b). ${ }^{7}$ However, these processes have very limited applications because the selectivity of the resulting products depends on aromatic substituents on the sulfur atom. ${ }^{7 b, f}$ One of the reasons why control of the selectivity of the ligand coupling is difficult is that conformation of sulfurane is not fixed through Berry's pseudorotation. ${ }^{7 a}$ To obtain unsymmetrical biaryls, previous ligand coupling was

\footnotetext{
${ }^{a}$ Department of Chemistry, Faculty of Science, Gakushuin University, 1-5-1 Mejiro, Toshima-ku, Tokyo 171-8588, Japan. E-mail: tatsuya.morofuji@gakushuin.ac.jp, naokazu.kano@gakushuin.ac.jp

${ }^{b}$ Department of Chemistry, Faculty of Science, Rikkyo University, 3-34-1 NishiIkebukuro, Toshima-ku, Tokyo 171-8501, Japan

$\dagger$ Electronic supplementary information (ESI) available: Experimental procedures, DFT calculations, and NMR data. See DOI: 10.1039/d0cc05830k
}

limited to use electron-poor heteroaromatic rings such as pyridine, azole, or quinoline as coupling partner, which promote desired ligand coupling. ${ }^{7 i, 8}$ In contrast, the selective synthesis of unsymmetrical biphenyl derivatives from sulfonium salts and aryl metals is a long-standing problem.

Herein, we report that $S$-arylphenothiazinium ions can solve the problem and be used as versatile arylation reagents for aryllithiums. The reaction of aryllithiums 1 with $S$-arylphenothiazinium ions 2 selectively afforded the corresponding biaryls 3 and phenothiazine (Fig. 1c). In contrast to the transition-metal-catalyzed approach,

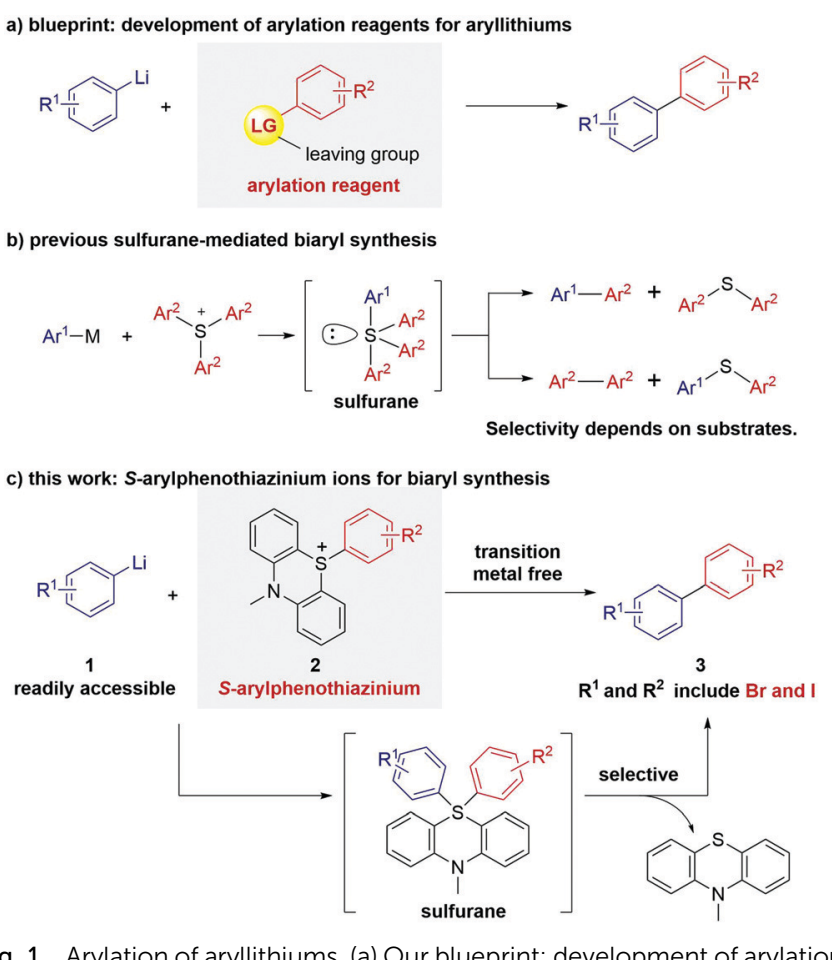

Fig. 1 Arylation of aryllithiums. (a) Our blueprint: development of arylation reagents for aryllithiums. (b) Previous work: reactivity of sulfurane and its limitation for the synthesis of biaryl. (c) This work: S-arylphenothiazinium ions used as electrophilic arylation reagents for synthesizing unsymmetrical biaryls. 
bromo- and iodo-substituted biaryls, which are attractive synthetic intermediates, could be synthesized using the present method. A computational study was performed to reveal the reaction mechanism of this sulfurane-mediated biaryl-formation.

First, we examined the reaction of some cyclic sulfonium ions $^{9}$ with phenyllithium (Table 1). Reaction of $S$-phenylphenothiazinium hexafluorophosphate (2a) with phenyllithium (1a) in THF at $-78{ }^{\circ} \mathrm{C}$ and $23{ }^{\circ} \mathrm{C}$ afforded biphenyl (3a) and $N$-methylphenothiazine (4) in good yields (entry 1). When the amount of 1a used was reduced, the conversion of 2a decreased (entry 2). In contrast, the reaction of $S$-phenylphenoxathiinium 5 with phenyllithium (1a) afforded a mixture of biphenyl (3a), phenoxathiin (6), and ring-opening product 7a (entry 3). As previously reported, the reaction of $S$-phenylthianthrenium 8 with phenyllithium (1a) under the same conditions afforded biphenyl (3a) in only $8 \%$ yield, while dibenzothiophene (9) (55\%), diphenyl sulfide (10) (51\%), and ring-opening product $7 \mathbf{b}(31 \%)$ were obtained in higher yields (entry 4$).{ }^{10}$ It was also reported that $S$-phenyldibenzothiophenium (11) afforded ring-opening product 7c in a quantitative yield (entry 5). ${ }^{11}$ It should be noted that ring opening product and $N$-methylcarbazole were not observed by ${ }^{1} \mathrm{H}$ NMR analysis when 2a was used. These results suggest that the phenothiazine moiety is essential for successful formation of biaryl. The specificity of $S$-phenylphenothiazinium was examined by theoretical calculations, as described later.

Table 1 Reaction of phenyllithium (1a) with sulfonium salts

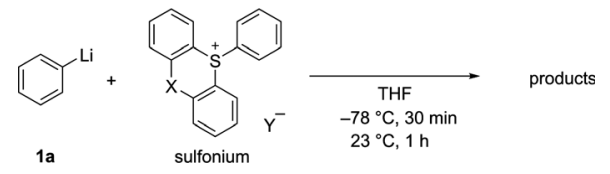

Entry Sulfonium Products
1<smiles>Cn1c2ccccc2c(=S)c2ccccc21</smiles><smiles>c1ccc(-c2ccccc2)cc1</smiles><smiles>CN1c2ccccc2Sc2ccccc21</smiles>

$2^{b}$ 2a

3<smiles>O=S(c1ccccc1)(c1ccccc1)=c1c2ccccc2oc2ccccc12</smiles>

3a $91 \%$

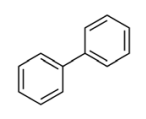

(N)

3a $38 \%{ }^{a}$<smiles>c1ccc(CSc2ccccc2[As]c2ccccc2)cc1</smiles><smiles>c1ccc(-c2ccccc2)cc1</smiles>

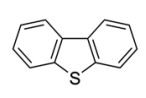

7 a $62 \%$

4

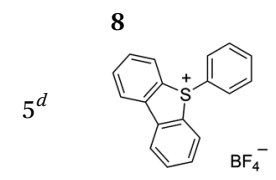

11

3a $8 \%^{a} \quad 955 \%^{c}$

$\Rightarrow$ Ph

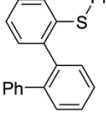

7c quant.
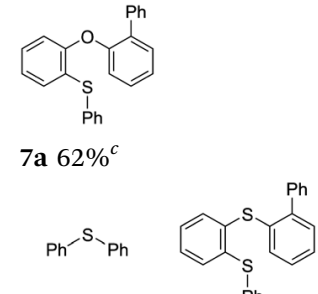

$\mathrm{Ph}$

Phenyllithium (1a) $(0.6 \mathrm{~mol})$ was added to sulfonium salt $(0.2 \mathrm{mmol})$ in THF $(5 \mathrm{~mL})$ at $-78{ }^{\circ} \mathrm{C}$. The reaction mixture was stirred at $-78{ }^{\circ} \mathrm{C}$ for $30 \mathrm{~min}$ and $23{ }^{\circ} \mathrm{C}$ for $1 \mathrm{~h} .{ }^{a}$ Determined by ${ }^{1} \mathrm{H}$ NMR spectroscopy.

${ }^{b} 0.4 \mathrm{mmol}$ of $1 \mathrm{a}$ was used. ${ }^{c}$ Isolated yield. ${ }^{d}$ Result from ref. 11.
The scope of the arylation of aryllithiums using $S$-arylphenothiazinium 2 investigated in the present study is shown in Scheme 1. To perform desymmetrizative mono-arylation of inexpensive 1,4-dibromobenzene, it was lithiated with $n$-BuLi and reacted

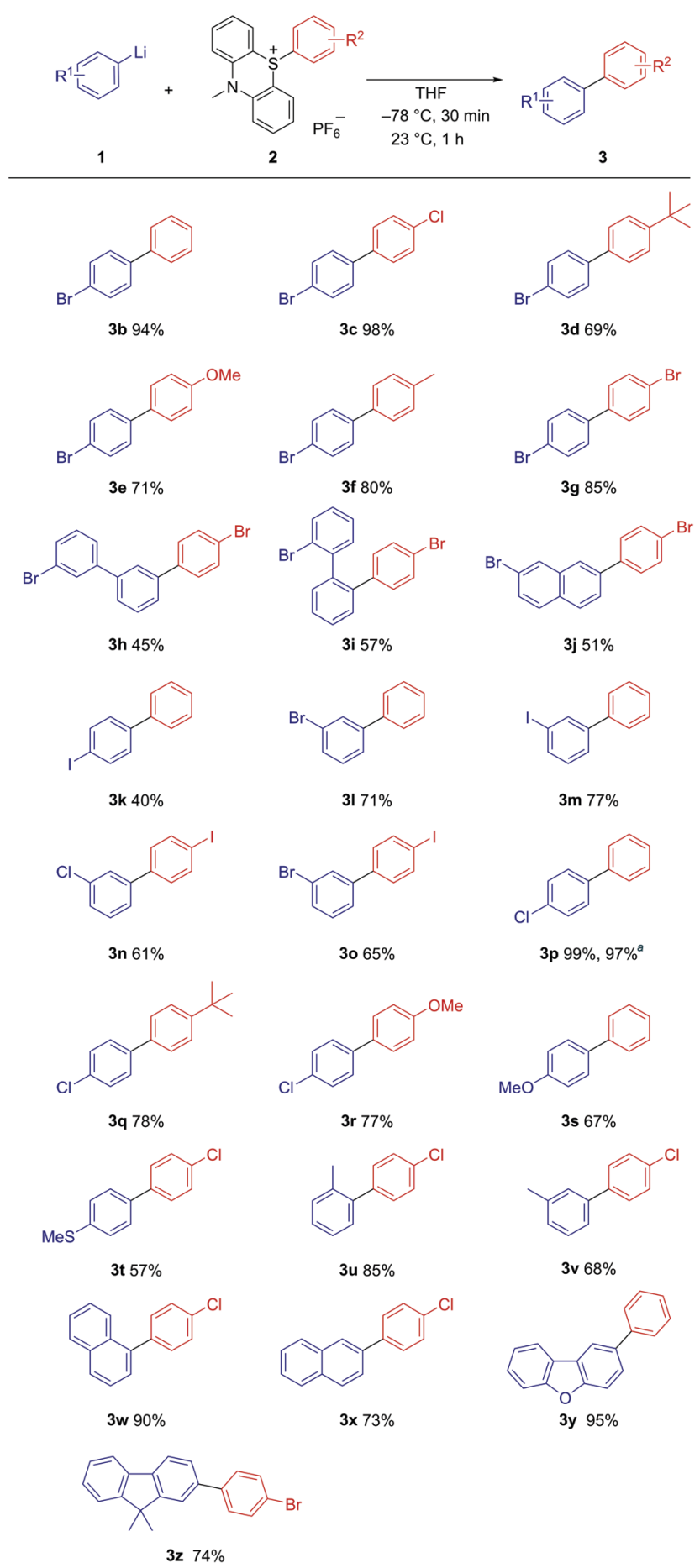

Scheme 1 Scope of cross-coupling of aryllithiums and S-arylphenothiazinium ions for synthesizing biaryls. S-Arylphenothiazinium $2(0.2 \mathrm{mmol})$ was added to a solution of aryllithium $1(0.6 \mathrm{mmol})$ in THF $(5 \mathrm{~mL})$ at $-78{ }^{\circ} \mathrm{C}$. The reaction mixture was stirred at $-78^{\circ} \mathrm{C}$ for $30 \mathrm{~min}$ and $23^{\circ} \mathrm{C}$ for $1 \mathrm{~h}$. Isolated yields of products are shown. See ESI, $\uparrow$ for details. ${ }^{2}$ The reaction was performed on a gram scale. 
with various $S$-arylphenothiaziniums successively to obtain the desired bromo-substituted biaryls (3b-3g) in good yields. Significantly, the reaction of $S$-(4-bromophenyl)phenothiazinium with aryllithiums bearing a bromo-substituent also afforded monoarylated products bearing two bromo groups, $\mathbf{3 h}, \mathbf{3 i}$, and $\mathbf{3} \mathbf{j}$. Other halogen-substituted biaryls could also be synthesized by the present method (3k-3o). The resulting bromo- or iodo-substituted biaryls are synthetically attractive intermediates for further derivatization by transition-metal-catalyzed cross-coupling reactions. It should be noted that bromo- or iodo-substituted biaryls have never been synthesized by Murahashi coupling because $\mathrm{C}-\mathrm{C}$ bond formation takes place at the halogen-substituted position. ${ }^{4}$ Furthermore, various unsymmetrical biaryls were synthesized using the present method $(\mathbf{3} \mathbf{p}-\mathbf{3 z})$. Reactions using $p$-chlorophenyllithium produced the desired products in good yields $(3 \mathbf{p}-3 \mathbf{r})$, whereas the use of aryllithiums bearing a methylchalcogeno group slightly decreased the yields of the product $(3 \mathbf{s}, 3 \mathbf{t})$.
Notably, the reaction efficiently produced $3 \mathbf{p}$ on the gram scale. Ortho- or meta-methyl- substituted aryllithium was applicable to the present reaction system $(3 \mathbf{u}, 3 \mathbf{v})$. Reactions of $\pi$-extended aryllithiums with $S$-arylphenothiaziniums also afforded the corresponding unsymmetrical biaryls in good yields (3w-3z).

To gain insight into the selective formation of unsymmetrical biaryls from the $S$-arylphenothiazinium ions, density functional theory (DFT) calculations were conducted at the B3LYP/6-311G+(d,p) level. Possible reaction pathways of phenyllithium(THF) $)_{3}$ (1a) with $S$-phenylphenothiazinium (2a) via the corresponding sulfurane were explored. The relative free energy profiles of the formation of biphenyl (path A), ringopening reaction (path $\mathbf{B}$ ), and formation of diphenyl sulfide (path C) from tetracoordinated sulfuranes are summarized in Fig. 2a. Three types of sulfurane intermediates (IM A, IM B, and IM C) afforded the corresponding transition states (TS A, TS B, and TS C). Each sulfurane intermediate has a different

\section{a)}

\begin{abstract}
path A: biphenyl formation path $\mathbf{B}$ : ring opening reaction

path $\mathbf{C}$ : diphenyl sulfide formation
\end{abstract}
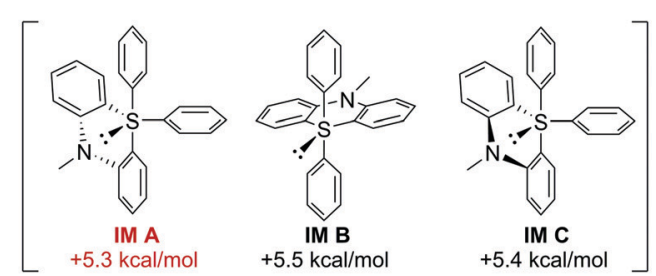

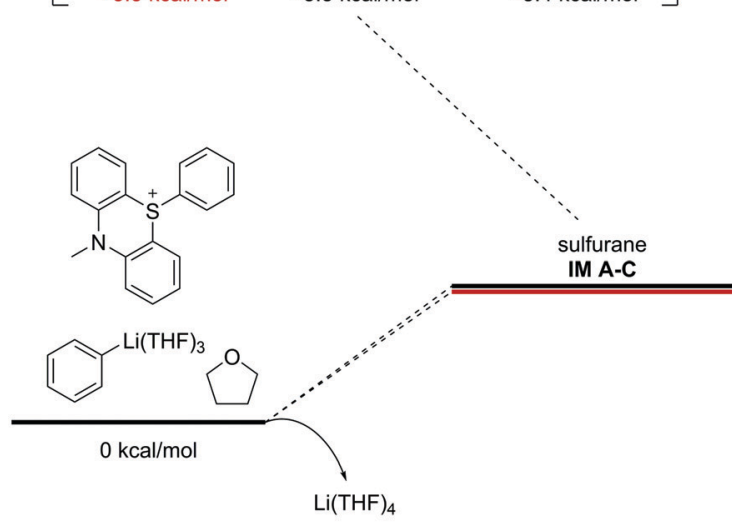

b)

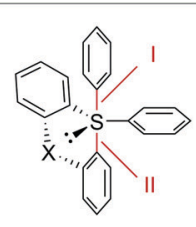

IM A $(X=N M e)$

IM D $(X=0)$

IM G $(X=S)$

IM J $\mathbf{J}=$ none $)$

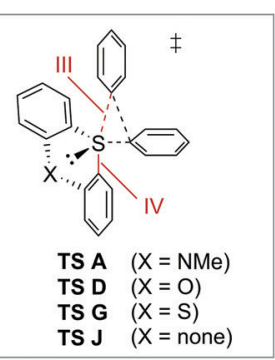

\begin{tabular}{ccccc}
\hline $\mathrm{X}$ & I & II & III & IV \\
\hline NMe & 2.20 & 1.97 & 2.65 & 1.81 \\
$\mathrm{O}$ & 2.11 & 2.04 & 2.63 & 1.82 \\
$\mathrm{~S}$ & 2.13 & 2.04 & 2.63 & 1.83 \\
none & 2.09 & 1.99 & 2.67 & 1.83 \\
\hline
\end{tabular}
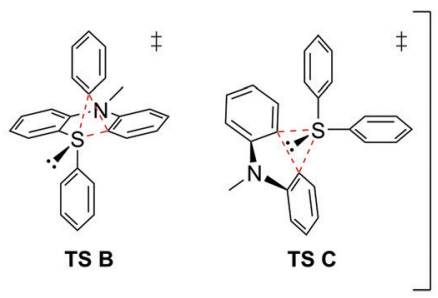

TS C: $+20.9 \mathrm{kcal} / \mathrm{mol}$

TS B: $+18.1 \mathrm{kcal} / \mathrm{mol}$

TS A: $+14.9 \mathrm{kcal} / \mathrm{mol}$
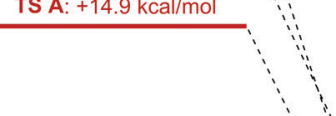

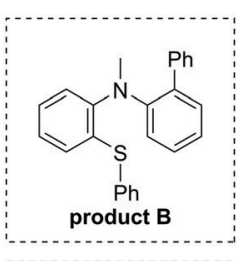

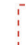

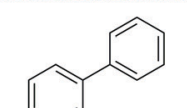

(1)

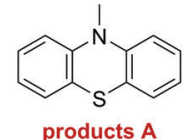

products $\mathrm{A}$

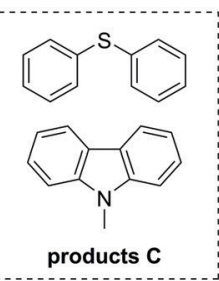

product B: $-74.5 \mathrm{kcal} / \mathrm{mol}$

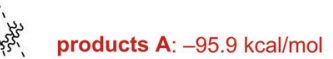

products C: $-106.8 \mathrm{kcal} / \mathrm{mol}$

c)

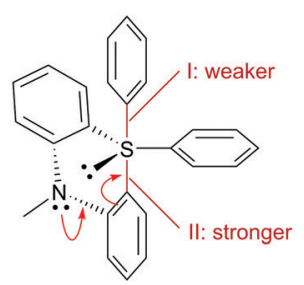

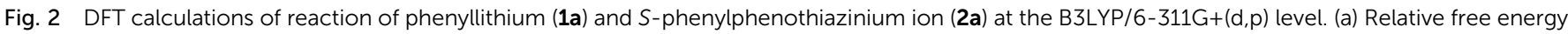

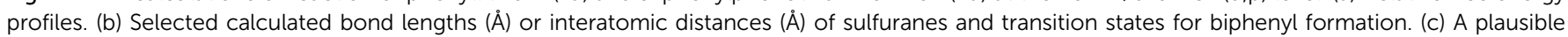
reason for the structural difference in sulfurane IM $\mathbf{A}$. 
pseudo-trigonal bipyramidal structure and similar free energy. Interconversion between IM A, IM B, and IM C occurs easily through pseudorotation. ${ }^{7 a}$ Therefore, the selectivity of the reaction products is determined by the $\mathbf{T S}$, not by the relative stability of IM. TS A is energetically more stable than TS B and TS C, which is in good agreement with the experimental results, as described in Table 1.

DFT calculations using $S$-phenylphenoxathiinium (4), $S$-phenylthianthrenium (6), or $S$-phenyldibenzothiophenium (7) instead of 2a indicated that biphenyl formation was not energetically preferred to ring opening-reaction and formation of diphenyl sulfide when 4, 6, or 7 was used (see ESI, $\dagger$ for details). These computational results are consistent with the experimental results. To understand the major factor in the preference of path A for $S$-phenylphenothiazinium ion, we compared the structural differences between IM A and TS A with their analogs (Fig. 2b). The bond lengths of apical bonds III and IV in each transition state are almost the same. In contrast, apical bonds I and II in IM A are longer and shorter, respectively, than those in IM D, IM G, and IM J. IM A has a relatively close structure to TS A. This indicates that biphenyl formation from the $S$-arylphenothiazinium ion (path A) requires a smaller structural distortion with a lower energy loss than that from other sulfonium ions. Such a structural difference is due to $\pi$-electron donation by the lone pair on the nitrogen atom, which would strengthen and shorten apical $\mathrm{C}-\mathrm{S}$ bond II (Fig. 2c). In general, when one apical bond is strengthened, the other apical bond becomes weaker and longer. ${ }^{12}$ Therefore, apical bond I in IM A is longer than that in others. This structural difference of IM A can be favorable for biaryl formation from phenyllithium (1a) and $S$-phenylphenothiazinium ion $\mathbf{2 a}$.

In summary, we developed $S$-arylphenothiazinium ions as efficient arylation reagents for aryllithiums. The reaction can provide various unsymmetrical biaryls without transition-metal catalysis. Notably, transformable halogen substituents such as bromo- and iodo-groups are tolerated. The resulting halogensubstituted biaryls are attractive synthetic intermediates. This result contrasts sharply with that obtained through palladiumcatalyzed Murahashi coupling. In contrast to other similar sulfonium salts, $S$-arylphenothiazinium ions are specifically suitable for biaryl synthesis through selective ligand coupling of a sulfurane intermediate, which is consistent with theoretical calculations. We are currently developing new transitionmetal-free arylation reactions using $S$-arylphenothiazinium ions; the results will be presented in due course.

This research was partially supported by the MEXTsupported program for the Strategic Research Foundation at Private Universities, and the Society of Iodine Science. DFT calculations were performed using Research Center for Computational Science, Okazaki, Japan.

\section{Conflicts of interest}

There are no conflicts to declare.

\section{Notes and references}

1 B. J. Wakefield, The Chemistry of Organolithium Compounds, Elsevier, Pergamon, 1974.

2 (a) N. Koike, T. Hattori, A. Takeda, Y. Okaishi and S. Miyano, Chem. Lett., 1997, 641; (b) J.-M. Becht, A. Gissot, A. Wagner and C. Mioskowski, Chem. - Eur. J., 2003, 9, 3209.

3 (a) J.-A. García-López and M. F. Greaney, Chem. Soc. Rev., 2016, 45, 6766; (b) F. Leroux and M. Schlosser, Angew. Chem., Int. Ed., 2002, 41, 4272; (c) T. Truong, M. Mesgar, K. K. A. Le and O. Daugulis, J. Am. Chem. Soc., 2014, 136, 8568.

4 (a) S. Murahashi, M. Yamamura, K. Yanagisawa, N. Mita and K. Kondo, J. Org. Chem., 1979, 44, 2408; (b) A. Nagaki, A. Kenmoku, Y. Moriwaki, A. Hayashi and J.-I. Yoshida, Angew. Chem., Int. Ed., 2010, 49, 7543; (c) M. Giannerini, M. Fananas-Mastral and B. L. Feringa, Nat. Chem., 2013, 5, 667; (d) E. B. Pinxterhuis, M. Giannerini, V. Hornillos and B. L. Feringa, Nat. Commun., 2016, 7, 11698; (e) D. Heijnen, F. Tosi, C. Vila, M. C. A. Stuart, P. H. Elsinga, W. Szymanski and B. L. Feringa, Angew. Chem., Int. Ed., 2017, 56, 3354.

5 For selected examples of transition-metal-free biaryl-synthesis, see (a) C.-L. Sun and Z.-J. Shi, Chem. Rev., 2014, 114, 9219; (b) T. Dohi, M. Ito, K. Morimoto, M. Iwata and Y. Kita, Angew. Chem., Int. Ed., 2008, 47, 1301; (c) T. Morofuji, A. Shimizu and J.-I. Yoshida, Angew. Chem., Int. Ed., 2012, 51, 7259; (d) E. Shirakawa, Y. Hayashi, K.-I. Itoh, R. Watabe, N. Uchiyama, W. Konagaya, S. Masui and T. Hayashi, Angew. Chem., Int. Ed., 2012, 51, 218; (e) M. Ito, H. Kubo, I. Itani, K. Morimoto, T. Dohi and Y. Kita, J. Am. Chem. Soc., 2013, 135, 14078; $(f)$ T. Yanagi, S. Otsuka, Y. Kasuga, K. Fujimoto, K. Murakami, K. Nogi, H. Yorimitsu and A. Osuka, J. Am. Chem. Soc., 2016, 138, 14582; $(g)$ M. C. Hilton, X. Zhang, B. T. Boyle, J. V. Alegre-Requena, R. S. Paton and A. McNally, Science, 2018, 362, 799; $(h)$ F. Kloss, T. Neuwirth, V. G. Haensch and C. Hertweck, Angew. Chem., Int. Ed., 2018, 57, 14476; (i) A. Music, A. N. Baumann, P. Spieß, A. Plantefol, T. C. Jagau and D. Didier, J. Am. Chem. Soc., $2020,142,4341$.

6 (a) W. A. Sheppard, J. Am. Chem. Soc., 1971, 93, 5597; (b) S. Ogawa, Y. Matsunaga, S. Sato, I. Iida and N. Furukawa, J. Chem. Soc., Chem. Commun., 1992, 1141.

7 (a) Ligand Coupling Reactions with Heteroatomic Compounds, ed. J.-P. Finet, Elsevier, Pergamon, 1998; (b) Y. H. Khim and S. Oae, Bull. Chem. Soc. Jpn., 1969, 42, 1968; (c) B. Trost, R. LaRochelle and R. Atkins, J. Am. Chem. Soc., 1969, 91, 2175; (d) R. W. LaRochelle and B. M. Trost, J. Am. Chem. Soc., 1971, 93, 6077; (e) B. M. Trost and H. C. Arndt, J. Am. Chem. Soc., 1973, 95, 5288; $(f)$ S. Oae, Croat. Chem. Acta, 1986, 59, 129; $(g)$ M. Hori, T. Kataoka, H. Shimizu, M. Ikemori and Y. Aoyama, J. Chem. Soc., Perkin Trans. 1, 1988, 1209; (h) S. Oae and Y. Uchida, Acc. Chem. Res., 1991, 24, 202; (i) S. Oae, H. Ishihara and M. Yoshihara, Chem. Heterocycl. Compd., 1995, 31, 917; $(j)$ S. Sato, O. Takahashi and N. Furukawa, Coord. Chem. Rev., 1998, 176, 483; (k) W. M. Dean, M. Šiaučiulis, T. E. Storr, W. Lewis and R. A. Stockman, Angew. Chem., Int. Ed., 2016, 55, 10013; (l) D. L. Chen, Y. Sun, M. Chen, X. Li, L. Zhang, X. Huang, Y. Bai, F. Luo and B. Peng, Org. Lett., 2019, 21, 3986.

8 (a) T. Kawai, N. Furukawa and S. Oae, Tetrahedron Lett., 1984, 25, 2549; (b) N. Furukawa, T. Shibutani and H. Fujihara, Tetrahedron Lett., 1987, 28, 5845; (c) M. Zhou, J. Tsien and T. Qin, Angew. Chem., Int. Ed., 2020, 59, 7372.

9 L. Racicot, T. Kasahara and M. A. Ciufolini, Org. Lett., 2014, 16, 6382. 10 B. Boduszek, H. J. Shine and T. K. Venkatachalam, J. Org. Chem., 1989, 54, 1616.

11 S. Sato and N. Furukawa, Tetrahedron Lett., 1995, 36, 2803.

12 L. J. Adzima, E. N. Duesler and J. C. Martin, J. Org. Chem., 1977, 42, 4001 . 